\title{
Iterative Schemes for Nonconvex Quasi-Variational Problems with $V$-Prox-Regular Data in Banach Spaces
}

\author{
M. Bounkhel ${ }^{1}$ and Dj. Bounekhel ${ }^{2}$ \\ ${ }^{1}$ Department of Mathematics, College of Science, King Saud University, P.O. Box 2455, Riyadh 11451, Saudi Arabia \\ ${ }^{2}$ Faculté des Sciences et Techniques, Université de Jijel, P.O. Box 98, Cité Ouled Aissa, Jijel, Algeria \\ Correspondence should be addressed to M. Bounkhel; bounkhel@ksu.edu.sa
}

Received 14 October 2016; Accepted 23 November 2016; Published 30 January 2017

Academic Editor: Adrian Petrusel

Copyright (c) 2017 M. Bounkhel and Dj. Bounekhel. This is an open access article distributed under the Creative Commons Attribution License, which permits unrestricted use, distribution, and reproduction in any medium, provided the original work is properly cited.

\begin{abstract}
In this paper, we propose an extension of quasi-equilibrium problems from the convex case to the nonconvex case and from Hilbert spaces to Banach spaces. The proposed problem is called quasi-variational problem. We study the convergence of some algorithms to solutions of the proposed nonconvex problems in Banach spaces.
\end{abstract}

\section{Introduction}

Let $X$ be a Banach space and let $X^{*}$ be the dual space of $X$. Let $\langle\cdot, \cdot\rangle$ denote the duality pairing of $X^{*}$ and $X$. Let $C: X \rightrightarrows X$ be a set-valued mapping with nonempty closed values and let $F: X \times X \rightarrow \mathbf{R}$ be a bifunction satisfying $F(x, x)=0$ for all $x \in \operatorname{Fix}(C):=\{x \in X: x \in C(x)\}$. We associate with a closed convex valued set-valued mapping $C$ and a convex bifunction $F$ the following well known quasi-equilibrium problem:

$$
\begin{aligned}
\text { Find } \quad & \bar{x} \in C(\bar{x}), \\
\text { such that } & F(\bar{x}, x) \geq 0, \quad(\operatorname{QEP}[C, F]) \\
& \forall x \in C(\bar{x}) .
\end{aligned}
$$

In this paper we propose the following appropriate extensions of (QEP $[C, F])$ from the convex case to the nonconvex case in Banach spaces setting. We associate with $C$ and $F$ the following nonconvex quasi-variational problem equilibrium problems:

$$
\begin{aligned}
\text { Find } & \bar{x} \in C(\bar{x}), \\
\text { s.t. } & {\left[-\partial^{\pi} F(\bar{x}, \cdot)(\bar{x})\right] \cap N^{\pi}(C(\bar{x}) ; \bar{x}) \quad(\operatorname{NQVP}[C, F]) } \\
& \neq \emptyset,
\end{aligned}
$$

where $\partial^{\pi}$ (resp. $N^{\pi}$ ) is the $V$-proximal subdifferential (resp. $V$-proximal normal cone) introduced and studied in [1].
The proposed nonconvex quasi-variational problem extends many existing quasi-equilibrium problems and quasi-variational inequalities from the convex case to the nonconvex case and from Hilbert spaces setting to Banach spaces setting.

(1) If $X$ is a Hilbert space, the proposed (NQVP $[C, F])$ becomes

Find $\bar{x} \in C(\bar{x})$,

such that $\left[-\partial^{P} F(\bar{x}, \cdot)(\bar{x})\right] \cap N^{P}(C(\bar{x}) ; \bar{x}) \neq \emptyset$,

where $\partial^{P}$ and $N^{P}$ are the usual proximal subdifferential and proximal normal cone in Hilbert spaces. This problem has been introduced and studied in Bounkhel et al. [2]. Since then it has been studied and extended in various ways in Hilbert spaces by the authors in [3] and in Noor [4] and many works (see for instances Noor et al. [5, 6]).

(2) If $X$ is a Hilbert space, $C$ is a convex closed set in $X, F$ is a convex bifunction, and $\rho=0$, then 
(NQVP $[C, F]$ ) becomes the following well known convex equilibrium problem:

$$
\begin{aligned}
\text { Find } & \bar{x} \in C, \\
\text { such that } & F(\bar{x}, x) \geq 0,
\end{aligned}
$$

$\forall x \in C$,

which has been studied in various works (see for instance Moudafi [7], M. A. Noor and K. I. Noor [5], and the references therein).

(3) If $F(x, y)=\langle T(x), y-x\rangle$, with $T: X \rightarrow X^{*}$, is a nonlinear operator then $(\mathrm{NQVP}[C, F])$ reduces to

$$
\begin{array}{ll}
\text { Find } & \bar{x} \in C(\bar{x}) \\
\text { s.t. } & -T(\bar{x}) \in N^{\pi}(C(\bar{x}) ; \bar{x})
\end{array}
$$

which will be shown in Section 4 to be equivalent in the uniform $V$-prox-regular case, for some $\rho \geq 0$, to the following quasi-variational inequality:

$$
\begin{aligned}
\text { Find } & \bar{x} \in C(\bar{x}), \\
\text { s.t. } & \langle T(\bar{x}), x-\bar{x}\rangle+\rho V(J(\bar{x}), x) \geq 0,
\end{aligned}
$$

$\forall x \in C(\bar{x})$.

This inequality is new in Banach spaces. However, it has been studied, in Hilbert spaces, in Bounkhel et al. [2], when $C$ is a uniformly $V$-prox-regular set (see also Bounkhel and Al-Sinan [8] and Noor et al. $[5,6])$.

When $\rho=0$ and $C(x) \equiv C$ the last inequality becomes

Find $\bar{x} \in C$,

such that $\langle T(\bar{x}), x-\bar{x}\rangle \geq 0$,

$\forall x \in C$,

which is known as the classical variational inequality introduced and studied in Stampacchia [9].

Our main objective of the present paper is to prove the convergence of some algorithms to solutions of the proposed nonconvex quasi-variational problem (NQVP $[C, F])$.

\section{Preliminaries}

In order to prepare the framework of our study we need to state some concepts and results. First we recall (see for instance $[1,10])$ the definition of $p$-uniformly convex and $q$ uniformly smooth Banach spaces. The space $X$ is said to be $p$-uniformly convex (resp. $q$-uniformly smooth) if there is a constant $c>0$ such that

$$
\delta_{X}(\epsilon) \geq c \epsilon^{p}\left(\operatorname{resp} \cdot \rho_{X}(t) \leq c t^{q}\right)
$$

where $\delta_{X}$ and $\rho_{X}$ are defined, respectively, by

$$
\begin{aligned}
& \delta_{X}(\epsilon)=\inf \left\{1-\left\|\frac{x+y}{2}\right\|:\|x\|=\|y\|=1,\|x-y\|\right. \\
& =\epsilon\}, \quad 0 \leq \epsilon \leq 2, \\
& \rho_{X}(t)=\sup \left\{\frac{1}{2}(\|x+y\|+\|x-y\|)-1:\|x\|=1,\|y\|\right. \\
& =t\}, \quad t>0 .
\end{aligned}
$$

Notice that the constants $p$ and $q$ in the previous definition always satisfy $p \geq 2$ and $q \in(1,2]$. Also we need to recall from [1] the concept of $V$-proximal subdifferential $\partial^{\pi} f(x)$ (called in [1] generalised proximal subdifferential). An element $x^{*} \in X^{*}$ belongs to $\partial^{\pi} f(x)$ provided that there exists $\sigma>0$ so that

$$
\left\langle x^{*}, x^{\prime}-x\right\rangle \leq f\left(x^{\prime}\right)-f(x)+\sigma V\left(J(x), x^{\prime}\right),
$$

for $x^{\prime}$ very close to $x$, where $J: X \rightarrow X^{*}$ is the normalised duality mapping and $V: X^{*} \times X \rightarrow \mathbf{R}$ is a functional defined by

$$
\begin{aligned}
& V\left(x^{*}, x\right)=\left\|x^{*}\right\|^{2}-2\left\langle x^{*}, x\right\rangle+\|x\|^{2}, \\
& \qquad \text { for any } x^{*} \in X^{*}, x \in X .
\end{aligned}
$$

For a closed nonempty set $S$ in $X$ and $\bar{x} \in S$, the authors in [1] defined the concept of $V$-proximal normal cone $N^{\pi}(S ; \bar{x})$ (called in [1] generalised proximal normal cone) by $N^{\pi}(S ; \bar{x})=$ $\partial^{\pi} \psi_{S}(\bar{x})$, where $\psi_{S}$ denotes the indicator function associated with $S$, that is, $\psi_{S}(x)=0$ if $x \in S$ and $\psi_{S}(x)=+\infty$ if $x \notin S$. We recall, respectively, the concepts of limiting Fréchet subdifferential $\partial^{L F}$ and limiting $V$-proximal subdifferential $\partial^{L \pi}$ (see [11]):

$$
\begin{aligned}
\partial^{L \pi} f(x) & =\limsup _{x^{\prime} \rightarrow x} \partial^{\pi} f\left(x^{\prime}\right) \\
& :=\left\{w-\lim _{n} x_{n}^{*}: x_{n}^{*} \in \partial^{\pi} f\left(x_{n}\right) \text { with } x_{n} \longrightarrow^{f} x\right\}, \\
\partial^{L F} f(x) & =\limsup _{x^{\prime} \rightarrow x} \partial^{F} f\left(x^{\prime}\right) \\
& :=\left\{w-\lim _{n} x_{n}^{*}: x_{n}^{*} \in \partial^{F} f\left(x_{n}\right) \text { with } x_{n} \longrightarrow^{f} x\right\},
\end{aligned}
$$

where $x_{n} \rightarrow^{f} x$ means $x_{n} \rightarrow x$ with $f\left(x_{n}\right) \rightarrow f(x)$ and

$$
\begin{gathered}
\partial^{F} f(x)=\left\{x^{*} \in X^{*}: \forall \epsilon>0, \exists \delta>0:\left\langle x^{*}, x^{\prime}-x\right\rangle\right. \\
\left.\leq f\left(x^{\prime}\right)-f(x)+\epsilon\left\|x^{\prime}-x\right\|, \forall x^{\prime} \in x+\delta \mathbf{B}\right\} .
\end{gathered}
$$

The limiting Fréchet normal cone is defined similarly, that is,

$$
\begin{aligned}
& \partial^{L F} N(S ; x)=\limsup _{x^{\prime} \rightarrow x} \partial^{F} N\left(S ; x^{\prime}\right) \\
& \quad:=\left\{w-\lim _{n} x_{n}^{*}: x_{n}^{*} \in N^{F}\left(S ; x_{n}\right) \text { with } x_{n} \longrightarrow^{S} x\right\},
\end{aligned}
$$


where $x_{n} \rightarrow^{S} x$ denotes $x_{n} \rightarrow x$ with $x_{n} \in S$ and $N^{F}(S ; x)$ is the Fréchet normal cone which is defined by $N^{F}(S ; \bar{x})=$ $\partial^{F} \psi_{S}(\bar{x})$.

These all nonconvex objects coincide with their analogues defined in Convex Analysis whenever the data are convex as the following proposition shows (see [1]).

Proposition 1. Let X be a reflexive Banach space.

(1) Let $f: X \rightarrow \mathbf{R} \cup+\infty$ be a l.s.c. convex function and $\bar{x} \in X$ with $f(\bar{x})<\infty$. Then

$$
\begin{aligned}
& \partial^{\pi} f(x)=\partial^{\text {Con. }} f(x):=\left\{x^{*} \in X^{*}:\left\langle x^{*} ; x-\bar{x}\right\rangle\right. \\
& \quad \leq f(x)-f(\bar{x}), \quad \forall x \in X\} .
\end{aligned}
$$

(2) Let $S$ be a closed convex subset in of $X$ and $\bar{x} \in S$. Then

$$
\begin{aligned}
N^{\pi}(S ; \bar{x}) & =N^{C o n .}(S ; \bar{x}) \\
& :=\left\{x^{*} \in X^{*}:\left\langle x^{*} ; x-\bar{x}\right\rangle \leq 0, \forall x \in S\right\} .
\end{aligned}
$$

The following result is needed in our study. It has been proved in [11].

Theorem 2. Let $X$ be a q-uniformly smooth and p-uniformly convex Banach space. Assume that $X$ admits an equivalent norm $\|\cdot\|$ such that $\|\cdot\|^{s}$ (for some $s \geq 2$ ) is $C^{2}$-differentiable on $X \backslash\{0\}$ and let $V$ be the functional associated with that norm $\|\cdot\|$.

(1) Let $f: X \rightarrow \mathbf{R} \cup\{\infty\}$, be a l.s.c. function at $\bar{x} \in \operatorname{dom} f$. Then

$$
\partial^{L \pi} f(\bar{x})=\partial^{F L} f(\bar{x}) .
$$

(2) Let $S$ be any closed nonempty set of $X$. Then

$$
N^{F L}(S ; \bar{x})=N^{L \pi}(S ; \bar{x}) .
$$

We notice that the class of spaces satisfying the assumptions of the previous theorem is very large; it contains obviously any Hilbert space and $L^{p}$ spaces and Sobolev spaces $W^{p, m}$ with $p \geq 2$ (see Theorem 1.1 in Section 5 in $[10,12]$ ) and for more examples and discussions we refer to [10, 12]. We close this section with the following two concepts of uniform $V$-prox-regularity for functions and sets (see [13]).

Definition 3. Let $X$ be a reflexive smooth Banach space. For a given $r \in(0, \infty]$, a subset $S$ is $V$-uniformly prox-regular with respect to $r$ provided that for all $x \in S$ and all nonzero $x^{*} \in N^{\pi}(S ; x)$ we have

$$
\left\langle\frac{x^{*}}{\left\|x^{*}\right\|}, x^{\prime}-x\right\rangle \leq \frac{1}{2 r} V\left(J(x), x^{\prime}\right), \quad \forall x^{\prime} \in S .
$$

We use the convention $1 / r=0$ for $r=+\infty$.

Obviously, this class contains the class of uniformly proxregular sets $([14,15])$ from Hilbert spaces to Banach spaces since in Hilbert spaces we have $V\left(J(x), x^{\prime}\right)=\left\|x-x^{\prime}\right\|^{2}$ and the $V$-proximal normal cone $N^{\pi}(S ; x)$ coincides with the usual proximal normal cone $N^{P}(S ; x)$.
Definition 4. Let $X$ be a reflexive smooth Banach space. Let $f: X \rightarrow \mathbf{R} \cup\{+\infty\}$ be a l.s.c. function and let $S \subset \operatorname{dom} f:=$ $\{x \in X: f(x)<\infty\}$ be a nonempty closed set in $X$. We recall from [13] that $f$ is said to be uniformly $V$-prox-regular over $S$ provided that for all $x \in S$ and all $x^{*} \in \partial^{\pi} f(x)$ we have

$$
\begin{array}{r}
\left\langle x^{*}, x^{\prime}-x\right\rangle \leq f\left(x^{\prime}\right)-f(x)+\frac{1}{2 r} V\left(J(x), x^{\prime}\right), \\
\forall x^{\prime} \in S .
\end{array}
$$

We say that $f$ is uniformly $V$-prox-regular around $\bar{x} \in$ $\operatorname{dom} f$ provided that $f$ is uniformly $V$-prox-regular over some closed neighborhood of $\bar{x}$; that is, there exists a closed neighborhood $V_{\bar{x}}$ of $\bar{x}$ such that $\forall x \in V_{\bar{x}}, \forall x^{*} \in \partial^{\pi} f(x)$ the inequality (18) holds for any $x^{\prime} \in V_{\bar{x}}$.

The following example is quoted from [13]. For its proof we refer the reader to [13].

Example 5. (1) Any l.s.c. proper convex function is uniformly $V$-prox-regular over any nonempty closed set $S$ in its domain with $r=+\infty$.

(2) Both the indicator function $\psi_{S}$ and the distance function $d_{S}$ of uniformly $V$-prox-regular set $S$ are uniformly $V$-prox-regular over $S$ with respect to the same constant $r$.

(3) Any lower- $C^{2}$ function $f$ over convex strongly compact $K$ in $X$ is uniformly $V$-prox-regular over $K$ with some $r \in(0,+\infty]$ (see [13] for the definition of lower- $C^{2}$ functions).

The following two lemmas are needed in our proofs in Section 4. The proof of the first one is proved in [1]. The second one is proved in [16].

Lemma 6. Let $X$ be a p-uniformly convex and q-uniformly smooth Banach space and $S$ be a bounded set. Then for some $\eta, \kappa>0$ we have

$$
\begin{aligned}
\eta^{-1}\|x-y\|^{p} \leq V(J(x), y) \leq \kappa^{-1}\|x-y\|^{q}, & \\
& \forall x, y \in S .
\end{aligned}
$$

Lemma 7. If $X$ is a uniformly convex Banach space, then the inequality

$$
V(J(x), y) \geq 8 C^{2} \delta_{X}\left(\frac{\|x-y\|}{4 C}\right)
$$

holds for all $x$ and $y$ in $X$, where $C=\sqrt{\left(\|x\|^{2}+\|y\|^{2}\right) / 2}$.

\section{Main Results}

First we show that in the convex case (NQVP $[C, F])$ coincides with the quasi-equilibrium problem (QEP $[C, F])$.

Proposition 8. Let $X$ be a reflexive Banach space. Assume that $C$ is a closed convex set-valued mapping and $F$ is a convex bifunction satisfying $F(x, x)=0$ for any $x \in \operatorname{Fix}(C)$. Then we have $(N Q V P[C, F]) \Leftrightarrow(Q E P[C, F])$. 
Proof.

$\Rightarrow$ ?. Let $\bar{x}$ be a solution of (NQVP $[C, F])$; that is, there exists $y^{*} \in \partial^{\pi} F(\bar{x}, \cdot)(\bar{x})$ such that $-y^{*} \in N^{\pi}(C(\bar{x}), \bar{x})$. Since $C(\bar{x})$ is a closed convex set, the $V$-proximal normal cone $N^{\pi}(C(\bar{x}), \bar{x})$ coincides with the convex normal cone $N^{\mathrm{Con} .}(C(\bar{x}), \bar{x})$ (by Proposition 1) and so

$$
\left\langle y^{*} ; x-\bar{x}\right\rangle \geq 0, \quad \forall x \in C(\bar{x}) .
$$

On the other hand, the convexity of the bifunction $F$ and Proposition 1 yield

$$
\left\langle y^{*} ; x-\bar{x}\right\rangle \leq F(\bar{x}, x)-F(\bar{x}, \bar{x}), \quad \forall x \in X .
$$

Since $\bar{x} \in C(\bar{x})$ we have $F(\bar{x}, \bar{x})=0$ (by assumption) and hence the previous two inequalities ensure

$$
F(\bar{x}, x) \geq 0, \quad \forall x \in C(\bar{x}) \text {; }
$$

that is, $\bar{x}$ is a solution of $(\mathrm{QEP}[C, F])$.

$\Leftarrow$ ?. Let $\bar{x}$ be a solution of $(\operatorname{NQEP}[C, F])$, that is, $F(\bar{x}, x) \geq$ $0, \forall x \in C(\bar{x})$. Since $C(\bar{x})$ is a closed convex set and $F(\bar{x}, \cdot)$ is a convex function, the function $x \mapsto h(x):=F(\bar{x}, x)+\psi_{C(\bar{x})}(x)$ admits at $\bar{x}$ a global minimum on $X$. It follows that

$$
\begin{aligned}
0 & \in \partial^{\text {Con. }} h(\bar{x})=\partial^{\text {Con. }} F(\bar{x}, \cdot)(\bar{x})+\partial^{\text {Con. }} \psi_{C(\bar{x})}(\bar{x}) \\
& =\partial^{\text {Con. }} F(\bar{x}, \cdot)(\bar{x})+N^{\text {Con. }}(C(\bar{x}) ; \bar{x}) .
\end{aligned}
$$

which is equivalent to $\left[-\partial^{\text {Con. }} F(\bar{x}, \cdot)(\bar{x})\right] \cap N^{\text {Con. }}(C(\bar{x}) ; \bar{x}) \neq$ $\emptyset$ and hence the proof is complete since $\partial^{\pi} F(\bar{x}, \cdot)(\bar{x})=$ $\partial^{\mathrm{Con} .} F(\bar{x}, \cdot)(\bar{x})$ and $N^{\pi}(C(\bar{x}), \bar{x})=N^{\mathrm{Con} .}(C(\bar{x}), \bar{x})$.

In the next proposition we establish an inequality characterisation of the proposed nonconvex quasi-variational problem (NQVP $[C, F]$ ) whenever the data $C$ and $F$ are uniformly $V$-prox-regular.

Proposition 9. Let $X$ be a reflexive Banach space and $\bar{x} \epsilon$ $X$. Assume that $C(\bar{x})$ is uniformly $V$-prox-regular with ratio $r \in(0, \infty]$ and that $F(\bar{x}, \cdot)$ is uniformly $V$-prox-regular over $C(\bar{x})$ with ratio $r^{\prime} \in(0, \infty]$. Assume also that $F(\bar{x}, \cdot)$ is $\gamma$ Lipschitz around $\bar{x}$ and $F(x, x)=0$ for any $x \in \operatorname{Fix}(C)$. If $\bar{x}$ is a solution of $(N Q V P[C, F])$, then $\bar{x}$ is a solution of the following nonconvex quasi-equilibrium problem. Find $\bar{x} \in C(\bar{x})$ such that

$$
F(\bar{x}, x)+\rho V(J \bar{x}, x) \geq 0, \quad \forall x \in C(\bar{x}), \quad(\mathrm{NQEP}[C, F])
$$

for some nonnegative $\rho \geq 0$.

Proof. Assume that $\bar{x}$ is a solution of (NQVP $[C, F])$; that is, $y^{*} \in \partial^{\pi} F(\bar{x}, \cdot)(\bar{x})$ such that $-y^{*} \in N^{\pi}(C(\bar{x}) ; \bar{x})$. By uniform $V$-prox-regularity of the set $C(\bar{x})$ we have

$$
\left\langle-y^{*}, x-\bar{x}\right\rangle \leq \frac{\left\|y^{*}\right\|}{2 r} V(J \bar{x}, x), \quad \forall x \in C(\bar{x}) .
$$

The $\gamma$-Lipschitz continuity of $F(\bar{x}, \cdot)$ ensures that $\left\|y^{*}\right\| \leq \gamma$ and so we obtain

$$
\left\langle-y^{*}, x-\bar{x}\right\rangle \leq \frac{\gamma}{2 r} V(J \bar{x}, x), \quad \forall x \in C(\bar{x}) .
$$

On the other hand the uniform $V$-prox-regularity of $F(\bar{x}, \cdot)$ over $C(\bar{x})$ with ratio $r^{\prime}>0$; we have

$$
\begin{aligned}
&\left\langle y^{*}, x-\bar{x}\right\rangle \leq \frac{1}{2 r^{\prime}} V(J \bar{x}, x)+F(\bar{x}, x)-F(\bar{x}, \bar{x}), \\
& \forall x \in C(\bar{x}) .
\end{aligned}
$$

Combining this inequality (27) with (26) we obtain

$$
\begin{array}{r}
F(\bar{x}, x)-F(\bar{x}, \bar{x})+\frac{1}{2 r^{\prime}} V(J \bar{x}, x) \geq-\frac{\gamma}{2 r} V(J \bar{x}, x) \\
\forall x \in C(\bar{x}) .
\end{array}
$$

Since $\bar{x} \in C(\bar{x})$ we have $F(\bar{x}, \bar{x})=0$ and so (28) becomes

$$
F(\bar{x}, x)+\rho V(J \bar{x}, x) \geq 0 \quad \forall x \in C(\bar{x}),
$$

with $\rho:=\gamma / 2 r+1 / 2 r^{\prime} \geq 0$. Thus the proof is complete.

It is a natural question to ask whether the converse in the previous proposition is true or not. The answer is positive provided that the space $X$ and the data $C$ and $F$ satisfy some additional assumptions as the following proposition shows.

Proposition 10. Let $X$ be a q-uniformly smooth and $p$ uniformly convex Banach space. Assume that $X$ admits an equivalent norm $\|\cdot\|$ such that $\|\cdot\|^{s}$ (for some $s \geq 2$ ) is $C^{2}$. differentiable on $X \backslash\{0\}$ and let $V$ be the functional associated with that norm $\|\cdot\|$. Assume that $C(\bar{x})$ is $V$-proximal normally regular at $\bar{x}$, that is, $N^{\pi}(C(\bar{x}), \bar{x})=N^{L \pi}(C(\bar{x}), \bar{x})$ and that $F(\bar{x}, \cdot)$ is $V$-proximal subdifferentially regular at $\bar{x}$, that is, $\partial^{\pi} F(\bar{x}, \cdot)(\bar{x})=\partial^{L \pi} F(\bar{x}, \cdot)(\bar{x})$. Assume that $F(x, x)=0$ for any $x \in \operatorname{Fix}(C)$. If $\bar{x}$ is a solution of $(N Q E P[C, F])$ for some $\rho \geq 0$, then $\bar{x}$ is a solution of $(N Q V P[C, F])$.

Proof. Let $\bar{x}$ be a solution of (NQEP $[C, F])$ for some $\rho \geq 0$; that is,

$$
F(\bar{x}, x)+\rho V(J \bar{x}, x) \geq 0 \quad \forall x \in C(\bar{x}) .
$$

Then $\bar{x}$ is a global minimum of the function $x \mapsto h(x)=$ $F(\bar{x}, x)+\rho V(J \bar{x}, x)+\psi_{C(\bar{x})}(x)$ over $X$ and hence

$$
\begin{aligned}
0 & \in \partial^{\pi} h(\bar{x}) \subset \partial^{L \pi} h(\bar{x}) \\
& =\partial^{L \pi}\left[F(\bar{x}, \cdot)+\rho V(J \bar{x}, \cdot)+\psi_{C(\bar{x})}(\cdot)\right](\bar{x}) .
\end{aligned}
$$

Note that the function $x \mapsto V(J(\bar{x}), x)$ is differentiable and its gradient is given by $\operatorname{grad}(V(J(\bar{x}), \cdot))(x)=2(J(x)-J(\bar{x}))$. Using the fact that the limiting $V$-proximal subdifferential 
coincides with the limiting Fréchet subdifferential (by Theorem 2) and the exact sum rules for the limiting Fréchet subdifferential (see for instance [17]) we can write

$$
\begin{aligned}
0 \in & \partial^{L \pi}\left[F(\bar{x}, \cdot)+\rho V(J \bar{x}, \cdot)+\psi_{C(\bar{x})}(\cdot)\right](\bar{x}) \\
\epsilon & \partial^{L F}\left[F(\bar{x}, \cdot)+\rho V(J \bar{x}, \cdot)+\psi_{C(\bar{x})}(\cdot)\right](\bar{x}) \\
\epsilon & \partial^{L F} F(\bar{x}, \cdot)(\bar{x})+\partial^{L F}(\rho V(J \bar{x}, \cdot))(\bar{x}) \\
& +\partial^{L F} \psi_{C(\bar{x})}(\cdot)(\bar{x}) \\
\epsilon & \partial^{L F} F(\bar{x}, \cdot)(\bar{x})+2 \rho(J(\bar{x})-J(\bar{x})) \\
& +N^{L F}(C(\bar{x}) ; \bar{x}) \\
\epsilon & \partial^{L F} F(\bar{x}, \cdot)(\bar{x})+N^{L F}(C(\bar{x}) ; \bar{x}) .
\end{aligned}
$$

This is equivalent to say that $\left[-\partial^{L F} F(\bar{x}, \cdot)(\bar{x})\right] \cap N^{L F}(C(\bar{x})$; $\bar{x}) \neq \emptyset$. Thus completing the proof since $\partial^{\pi} F(\bar{x}, \cdot)(\bar{x})=$ $\partial^{L \pi} F(\bar{x}, \cdot)(\bar{x})=\partial^{L F} F(\bar{x}, \cdot)(\bar{x})$ and $N^{\pi}(C(\bar{x}), \bar{x})=N^{L \pi}(C(\bar{x})$; $\bar{x})=N^{L F}(C(\bar{x}) ; \bar{x})$.

The following proposition has its own interest and is needed to prove the equivalence between (NQVP $[C, F])$ and $(\mathrm{NQEP}[C, F])$ whenever $C$ and $F$ are uniformly $V$-proxregular.

Proposition 11. Let $X$ be a reflexive Banach space and let $f: X \rightarrow \mathbf{R} \cup\{\infty\}$ be a l.s.c. function and let $\bar{x} \in \operatorname{dom} f$. If $f$ is uniformly $V$-prox-regular around $\bar{x}$, then $\partial^{\pi} f(\bar{x})=$ $\partial^{L \pi} f(\bar{x})$; that is, $f$ is $V$-proximal subdifferentially regular at $\bar{x}$. Consequently, for any uniformly $V$-prox-regular closed set $S$ at $\bar{x} \in S$ we have $N^{\pi}(S, \bar{x})=N^{L \pi}(S ; \bar{x})$; that is, $S$ is $V$-proximal normally regular at $\bar{x}$.

Proof. We only prove the first assertion; the second one follows directly from the first one and Example 5 Part (2). Since we always have the inclusion $\partial^{\pi} f(\bar{x}) \subset \partial^{L \pi} f(\bar{x})$, it is enough to prove the reverse one, that is, $\partial^{L \pi} f(\bar{x}) \subset \partial^{\pi} f(\bar{x})$. Let $x^{*} \in \partial^{L \pi} f(\bar{x})$; that is, there exists $x_{n} \rightarrow^{f} x$ and $x_{n}^{*} \in$ $\partial^{\pi} f\left(x_{n}\right)$ such that $x^{*}=w-\lim _{n} x_{n}^{*}$. By the uniform $V$-proxregularity of $f$ around $\bar{x}$, there exist $r>0$ and $\delta>0$ such that for any $x \in \bar{x}+\delta \mathbf{B}$ and any $y^{*} \in \partial^{\pi} f(x)$

$$
\begin{aligned}
\left\langle y^{*}, x^{\prime}-x\right\rangle \leq \frac{1}{2 r} V\left(J x, x^{\prime}\right)+f\left(x^{\prime}\right)- & f(x), \\
& \forall x^{\prime} \in x+\delta \mathbf{B} .
\end{aligned}
$$

Since $x_{n} \rightarrow \bar{x}$ we can write for $n$ large enough that $x_{n} \in \bar{x}+$ $(\delta / 2) \mathbf{B}$ and hence by (33) we have

$$
\begin{array}{r}
\left\langle x_{n}^{*}, x^{\prime}-x_{n}\right\rangle \leq \frac{1}{2 r} V\left(J x_{n}, x^{\prime}\right)+f\left(x^{\prime}\right)-f\left(x_{n}\right), \\
\forall x^{\prime} \in x_{n}+\delta \mathbf{B} .
\end{array}
$$

Fix any $y \in \bar{x}+(\delta / 2) \mathbf{B}$. Clearly $y \in x_{n}+(\delta / 2) \mathbf{B}+(\delta / 2) \mathbf{B} \subset$ $x_{n}+\delta \mathbf{B}$ and hence (34) ensures

$$
\begin{aligned}
\left\langle x^{*}, y-\bar{x}\right\rangle= & \left\langle x^{*}-x_{n}^{*}, y-\bar{x}\right\rangle+\left\langle x_{n}^{*}, y-x_{n}\right\rangle \\
& +\left\langle x_{n}^{*} ; x_{n}-\bar{x}\right\rangle \\
\leq & \left\langle x^{*}-x_{n}^{*}, y-\bar{x}\right\rangle+\left\langle x_{n}^{*} ; x_{n}-\bar{x}\right\rangle \\
& +\frac{1}{2 r} V\left(J x_{n}, y\right)+f(y)-f\left(x_{n}\right) .
\end{aligned}
$$

Using now the fact that $x_{n} \rightarrow f \bar{x}$, the continuity of $J$ and $V$, and the weak convergence of $x_{n}^{*}$ to $x^{*}$ to pass to the limit as $n$ goes to $\infty$ and to get

$$
\left\langle x^{*}, y-\bar{x}\right\rangle \leq \frac{1}{2 r} V(J \bar{x}, y)+f(y)-f(\bar{x}),
$$

for any $y \in \bar{x}+(\delta / 2) \mathbf{B}$, this means by definition that $x^{*} \epsilon$ $\partial^{\pi} f(\bar{x})$ and the proof is complete.

Using this result together with Propositions 9 and 10 we obtain the equivalence between (NQVP $[C, F])$ and (NQEP $[C, F])$.

Proposition 12. Let $X$ be a q-uniformly smooth and $p$ uniformly convex Banach space and $\bar{x} \in X$. Assume that $X$ admits an equivalent norm $\|\cdot\|$ such that $\|\cdot\|^{s}$ (for some $s \geq 2$ ) is $C^{2}$-differentiable on $X \backslash\{0\}$ and let $V$ be the functional associated with that norm $\|\cdot\|$. Assume that $C(\bar{x})$ is uniformly $V$-prox-regular with ratio $r \in(0, \infty]$ and that $F(\bar{x}, \cdot)$ is uniformly $V$-prox-regular over $C(\bar{x})$ with ratio $r^{\prime} \in(0, \infty]$. Assume also that $F(\bar{x}, \cdot)$ is $\gamma$-Lipschitz around $\bar{x}$ and $F(x, x)=$ 0 for any $x \in \operatorname{Fix}(C)$. Then $(N Q V P[C, F])$ is equivalent to $(N Q E P[C, F])$ for some $\rho \geq 0$.

\section{Convergence Analysis}

4.1. Case 1: C Is a Constant Set-Valued Mapping. In this case the proposed problem becomes as follows:

$$
\begin{array}{ll}
\text { Find } & \bar{x} \in C \\
\text { s.t. } & {\left[-\partial^{\pi} F(\bar{x}, \cdot)(\bar{x})\right] \cap N^{\pi}(C ; \bar{x}) \neq \emptyset .}
\end{array}
$$

In this subsection we propose the following algorithm.

Algorithm 13. Let $\rho \geq 0$ and $\lambda_{n}>0$ for all $n \geq 1$;

(1) Select $x_{0} \in C$;

(2) For $n \geq 1$ select $x_{n+1} \in C$ such that

$$
\begin{aligned}
& \lambda_{n}^{-1}\left\langle J\left(x_{n}\right)-J\left(x_{n+1}\right), x-x_{n+1}\right\rangle \\
& \quad \leq F\left(x_{n}, x\right)+\rho V\left(J\left(x_{n}\right), x\right), \quad \forall x \in C .
\end{aligned}
$$

Theorem 14. Let $X$ be a q-uniformly convex Banach space. Let $C$ be a closed nonempty subset of $X$ and let $F: C \times C \rightarrow \mathbf{R}$ be a bifunction satisfying $F(x, x)=0$ for any $x \in \operatorname{Fix}(C)$. Let $\left\{x_{n}\right\}_{n}$ be a sequence generated by Algorithm 13. Assume that 
(1) $C$ is V-uniformly prox-regular with some $r \in(0, \infty]$;

(2) $C$ is ball compact; that is, $C \cap \eta B$ is compact for any $\eta>0$

(3) The solution set of $(N Q V P[C, F])$ is nonempty;

(4) $F$ is $W$-strongly monotone over $C$ for some $\sigma \geq 0$; that is,

$F(x, y)+F(y, x) \leq-\sigma W(x, y), \quad \forall x, y \in C$,

where $W(x, y):=(1 / 2)[V(J(x), y)+V(J(y), x)]$;

(5) $F$ is upper semicontinuous with respect to the first variable over $C$; that is,

$$
\limsup _{x^{\prime} \rightarrow x} F\left(x^{\prime}, y\right) \leq F(x, y) \quad \forall x, y \in C ;
$$

(6) The bifurcation $F$ is $\gamma$-Lipschitz with respect to the second variable and $F\left(x_{n+1}, \cdot\right)$ is $V$-uniformly proxregular over $C$ with some $r^{\prime} \in(0,+\infty]$;

(7) There exists $\lambda>0$ such that $\lambda_{n} \geq \lambda$ for all $n$;

(8) The parameters $r, r^{\prime}, \gamma, \rho, \sigma$ satisfy the inequalities $2 \rho \leq$ $\gamma / 2 r+1 / 2 r^{\prime} \leq \sigma / 3$.

Then, there exists subsequence of $\left\{x_{n}\right\}$ converges to $\tilde{x} \in C$ which solves $(N V P[C, F])$.

Proof. Let $\bar{x} \in C$ be a solution of $(\operatorname{NVP}[C, F])$. Then by Proposition 9 we have

$$
F(\bar{x}, x)+\rho_{0} V(J(\bar{x}), x) \geq 0, \quad \forall x \in C,
$$

for $\rho_{0}:=\gamma / 2 r+1 / 2 r^{\prime}$. By the $W$-strong monotonicity of $F$ over $C$ we have

$$
F(x, \bar{x})+F(\bar{x}, x) \leq-\sigma W(x, \bar{x}), \quad \forall x \in C .
$$

By setting $x=x_{n}$ in these two inequalities we get

$$
\begin{aligned}
F\left(x_{n}, \bar{x}\right)+F\left(\bar{x}, x_{n}\right) & \leq-\sigma W\left(x_{n}, \bar{x}\right), \\
-F\left(\bar{x}, x_{n}\right) & \leq \rho_{0} V\left(J(\bar{x}), x_{n}\right) .
\end{aligned}
$$

Combining these two inequalities we obtain

$$
\begin{aligned}
F\left(x_{n}, \bar{x}\right) & \leq \rho_{0} V\left(J(\bar{x}), x_{n}\right)-\sigma W\left(x_{n}, \bar{x}\right) \\
& \leq\left(2 \rho_{0}-\sigma\right) W\left(x_{n}, \bar{x}\right) .
\end{aligned}
$$

Using the 8 th assumption in Theorem 14 we have $2 \rho_{0}-\sigma \leq$ $-\rho_{0}$ and hence

$$
F\left(x_{n}, \bar{x}\right) \leq-\rho_{0} W\left(x_{n}, \bar{x}\right) .
$$

This combined with Algorithm 13 gives

$$
\begin{aligned}
\left\langle x_{n+1}^{*}, \bar{x}-x_{n+1}\right\rangle & \leq F\left(x_{n}, \bar{x}\right)+\rho V\left(J\left(x_{n}\right), \bar{x}\right) \\
& \leq-\rho_{0} W\left(x_{n}, \bar{x}\right)+\rho V\left(J\left(x_{n}\right), \bar{x}\right) \\
& \leq\left(2 \rho-\rho_{0}\right) W\left(x_{n}, \bar{x}\right),
\end{aligned}
$$

with $x_{n+1}^{*}:=\lambda_{n}^{-1}\left[J\left(x_{n}\right)-J\left(x_{n+1}\right)\right]$. Therefore,

$$
\begin{gathered}
\left\langle J\left(x_{n}\right)-J\left(x_{n+1}\right), \bar{x}-x_{n+1}\right\rangle \\
\leq \lambda_{n}\left(2 \rho-\rho_{0}\right) W\left(x_{n}, \bar{x}\right) .
\end{gathered}
$$

Define now a sequence of nonnegative real numbers $\phi_{n}=$ $(1 / 2) V\left(J\left(x_{n}\right), \bar{x}\right)$. It is not hard to verify that

$$
\begin{aligned}
& 2\left[\phi_{n+1}-\phi_{n}\right]+V\left(J\left(x_{n}\right), x_{n+1}\right) \\
& \quad=2\left\langle J\left(x_{n}\right)-J\left(x_{n+1}\right), \bar{x}-x_{n+1}\right\rangle .
\end{aligned}
$$

Indeed,

$$
\begin{aligned}
2\left[\phi_{n+1}-\phi_{n}\right]=V\left(J\left(x_{n+1}\right), \bar{x}\right)-V\left(J\left(x_{n}\right), \bar{x}\right) \\
=\left[\left\|J\left(x_{n+1}\right)\right\|^{2}-2\left\langle J\left(x_{n+1}\right), \bar{x}\right\rangle+\|\bar{x}\|^{2}\right] \\
\quad-\left[\left\|J\left(x_{n}\right)\right\|^{2}-2\left\langle J\left(x_{n}\right), \bar{x}\right\rangle+\|\bar{x}\|^{2}\right] \\
=\quad\left\|J\left(x_{n+1}\right)\right\|^{2}+2\left\langle J\left(x_{n}\right)-J\left(x_{n+1}\right), \bar{x}\right\rangle \\
\quad-\left\|J\left(x_{n}\right)\right\|^{2} \\
=2\left\langle J\left(x_{n}\right)-J\left(x_{n+1}\right), \bar{x}\right\rangle-\left\|J\left(x_{n+1}\right)\right\|^{2} \\
\quad-\left\|J\left(x_{n}\right)\right\|^{2}+2\left\langle J\left(x_{n+1}\right), x_{n+1}\right\rangle \\
=2\left\langle J\left(x_{n}\right)-J\left(x_{n+1}\right), \bar{x}\right\rangle-V\left(J\left(x_{n}\right), x_{n+1}\right) \\
\quad-2\left\langle J\left(x_{n}\right), x_{n+1}\right\rangle+2\left\langle J\left(x_{n+1}\right), x_{n+1}\right\rangle \\
=2\left\langle J\left(x_{n}\right)-J\left(x_{n+1}\right), \bar{x}\right\rangle-V\left(J\left(x_{n}\right), x_{n+1}\right) \\
\quad-2\left\langle J\left(x_{n}\right)-J\left(x_{n+1}\right), x_{n+1}\right\rangle \\
=2\left\langle J\left(x_{n}\right)-J\left(x_{n+1}\right), \bar{x}-x_{n+1}\right\rangle \\
-
\end{aligned}
$$

It follows that

$$
\phi_{n+1}-\phi_{n} \leq\left\langle J\left(x_{n}\right)-J\left(x_{n+1}\right), \bar{x}-x_{n+1}\right\rangle,
$$

which ensures with (46) that

$$
\phi_{n+1}-\phi_{n} \leq \lambda_{n}\left(2 \rho-\rho_{0}\right) W\left(x_{n}, \bar{x}\right) .
$$

Using the assumption $\rho_{0} \geq 2 \rho$ in the 8 th assumption we obtain

$$
\phi_{n+1} \leq \phi_{n}
$$

Therefore, the sequence $\left\{\phi_{n}\right\}$ is a nonincreasing converging sequence to some limit and so it is bounded by some $\alpha>0$. Thus by the properties of the functional $V$ we obtain

$$
\left(\|\bar{x}\|-\left\|x_{n}\right\|\right)^{2} \leq V\left(J\left(x_{n}\right), \bar{x}\right)=2 \phi_{n} \leq 2 \alpha
$$

and so

$$
\left\|x_{n}\right\| \leq\|\bar{x}\|+\sqrt{2 \alpha}
$$


that is, $\left\{x_{n}\right\}$ is bounded and so by the $q^{\prime}$-uniform convexity of $X^{*}$ (by Lemma 6) we have for some $\eta>0$ depending on $\alpha$ and on the space $X^{*}$ the inequality

$$
\begin{aligned}
\left\|J\left(x_{n+1}\right)-J\left(x_{n}\right)\right\|^{q^{\prime}} & \leq \eta V_{*}\left(J^{*}\left(J\left(x_{n+1}\right)\right), J\left(x_{n}\right)\right) \\
& =\eta V\left(J\left(x_{n}\right), x_{n+1}\right)
\end{aligned}
$$

where $J^{*}: X^{*} \rightarrow X^{* *}(=X)$ is the normalised duality mapping on $X^{*}$ and $V_{*}: X^{* *} \times X^{*} \rightarrow \mathbf{R}$ is the functional defined by

$$
\begin{aligned}
& V_{*}\left(x^{* *} ; x^{*}\right):=\left\|x^{* *}\right\|^{2}-2\left\langle x^{* *} ; x^{*}\right\rangle+\left\|x^{*}\right\|^{2}, \\
& \forall x^{*} \in X^{*}, x^{* *} \in X^{* *} .
\end{aligned}
$$

Using now (46) and (47) and the assumption $\rho_{0} \geq 2 \rho$ we obtain

$$
\frac{1}{2} V\left(J\left(x_{n}\right), x_{n+1}\right) \leq \phi_{n}-\phi_{n+1} .
$$

Therefore, it follows from the 7th assumption of Theorem 14 that

$$
\begin{aligned}
\left\|x_{n+1}^{*}\right\|^{q^{\prime}} & =\lambda_{n}^{-q^{\prime}}\left\|J\left(x_{n+1}\right)-J\left(x_{n}\right)\right\|^{q^{\prime}} \\
& \leq \lambda^{-q^{\prime}}\left\|J\left(x_{n+1}\right)-J\left(x_{n}\right)\right\|^{q^{\prime}} \\
& \leq \lambda^{-q^{\prime}} \eta V\left(J\left(x_{n}\right), x_{n+1}\right) \\
& \leq \frac{2 \eta}{\lambda q^{\prime}}\left[\phi_{n}-\phi_{n+1}\right] \longrightarrow 0 \text { as } n \longrightarrow \infty,
\end{aligned}
$$

which ensures that $\lim _{n \rightarrow \infty} x_{n+1}^{*}=0$. On the other hand, since $\left\{x_{n}\right\}$ is bounded in $C$ and $C$ is ball compact then there exists a subsequence $\left\{x_{n_{k}}\right\}$ which converges to some limit $\tilde{x} \in C$. By Algorithm 13 this subsequence satisfies

$$
\begin{array}{r}
\left\langle x_{n_{k}+1}^{*}, x-x_{n_{k}+1}\right\rangle \leq F\left(x_{n_{k}}, x\right)+\rho V\left(J\left(x_{n_{k}}\right), x\right), \\
\forall k, \forall x \in C .
\end{array}
$$

Thus, by letting $k \rightarrow \infty$ in the inequality (58) and by taking into account the upper semicontinuity of $F$ and the continuity of $V$ and $J$, we obtain

$$
0 \leq F(\tilde{x}, x)+\rho V(J(\tilde{x}), x), \quad \forall x \in C .
$$

This means that $\tilde{x}$ is a solution of $(\operatorname{NEP}[C, F])$. Finally, using now Proposition 12 we get $\tilde{x}$ is a solution of $(\mathrm{NVP}[C, F])$ and so the proof is complete.

4.2. Case 2: C Is a General Set-Valued Mapping. In this general case we propose the following algorithm.

Algorithm 15. Let $\rho \geq 0$ and $\lambda_{n}>0$ for all $n \geq 1$;

(1) Select $x_{0} \in C\left(x_{0}\right)$;

(2) For $n \geq 1$ select $x_{n+1} \in C\left(x_{n}\right)$ such that

$$
\begin{aligned}
& \lambda_{n}^{-1}\left\langle J\left(x_{n}\right)-J\left(x_{n+1}\right), x-x_{n+1}\right\rangle \\
& \quad \leq F\left(x_{n}, x\right)+\rho V\left(J\left(x_{n}\right), x\right), \quad \forall x \in \operatorname{Im} C,
\end{aligned}
$$

where $M>0$ is a given positive number and $\operatorname{Im} C$ is the image of $C$, that is, $\operatorname{Im} C:=\{y \in X: \exists x \in$ $X$ such that $y \in C(x)\}$.

Obviously Algorithm 15 coincides with Algorithm 13 when $C$ is a constant set-valued mapping. However the assumptions assumed on $F$ in the previous subsection are not sufficient to prove the convergence of the sequence $\left\{x_{n}\right\}$ generated by Algorithm 15 to a solution of (NQVP $[C, F])$. We need to replace the $W$-strong monotonicity by a relaxed $W$-strong monotonicity of the bifunction $F$ over $\operatorname{Im} C$ and we do not assume the nonemptiness of the solution set of the proposed problem. We will say that $F$ is relaxed $W$-strongly monotone over Im $C$ provided that for some $\sigma \geq 0$ we have

$$
F(x, y) \leq-\sigma W(x, y), \quad \forall x, y \in \operatorname{Im} C .
$$

By symmetry of $W$, it is clear that any $W$-relaxed strongly monotone bifunction with respect to $\sigma \geq 0$ is $W$-strongly monotone with respect to $2 \sigma$. This relaxed assumption on $F$ has been used in Hilbert spaces in [4] and in Banach spaces in [13]. The following theorem is our main result in this subsection.

Theorem 16. Let $X$ be a q-uniformly convex Banach space. Let $C$ be a closed nonempty subset of $X$ and let $F: C \times C \rightarrow \mathbf{R}$ be a bifunction satisfying $F(x, x)=0$ for any $x \in \operatorname{Fix}(C)$. Let $\left\{x_{n}\right\}_{n}$ be a sequence generated by Algorithm 15. Assume that

(1) The values of $C$ are $V$-uniformly prox-regular with some ratio $r \in(0, \infty)$;

(2) The image of $C$ is ball compact in $X$ and its graph is closed;

(3) $F$ is relaxed $W$-strongly monotone over Im $C$ with some $\sigma>0$

(4) $F$ is upper semicontinuous with respect to the first variable over $\operatorname{Im} C$;

(5) $F\left(x_{n}, \cdot\right)$ is $V$-uniformly prox-regular over $\operatorname{Im} C$ with some $r^{\prime} \in(0,+\infty]$;

(6) There exists $\lambda>0$ such that $\lambda_{n} \geq \lambda$ for all $n$;

(7) The nonnegative parameter $\rho$ is taken in the interval $[0, \sigma / 2]$.

Then, there exists subsequence of $\left\{x_{n}\right\}$ converging to a solution of $(N Q V P[C, F])$.

Proof. Let $\bar{x} \in \operatorname{Im} C$. By the relaxed $W$-strong monotonicity of $F$ over $\operatorname{Im} C$ we have

$$
F\left(x_{n}, \bar{x}\right) \leq-\sigma W\left(x_{n}, \bar{x}\right), \quad \forall n \geq 1 .
$$

By Algorithm 15 we have

$$
\left\langle x_{n+1}^{*}, \bar{x}-x_{n+1}\right\rangle \leq F\left(x_{n}, \bar{x}\right)+\rho V\left(J\left(x_{n}\right), \bar{x}\right),
$$

with $x_{n+1}^{*}:=\lambda_{n}^{-1}\left[J\left(x_{n}\right)-J\left(x_{n+1}\right)\right]$. Combining these two inequalities we get

$$
\begin{aligned}
\left\langle x_{n+1}^{*}, \bar{x}-x_{n+1}\right\rangle & \leq \rho V\left(J\left(x_{n}\right), \bar{x}\right)-\sigma W\left(x_{n}, \bar{x}\right) \\
& \leq(2 \rho-\sigma) W\left(x_{n}, \bar{x}\right) .
\end{aligned}
$$


Therefore,

$$
\begin{gathered}
\left\langle J\left(x_{n}\right)-J\left(x_{n+1}\right), \bar{x}-x_{n+1}\right\rangle \\
\leq \lambda_{n}(2 \rho-\sigma) W\left(x_{n}, \bar{x}\right) .
\end{gathered}
$$

Define now the same nonnegative real sequence $\phi_{n}=$ $(1 / 2) V\left(J\left(x_{n}\right), \bar{x}\right)$ used in the proof of Theorem 14. Then we have

$$
\phi_{n+1}-\phi_{n} \leq\left\langle J\left(x_{n}\right)-J\left(x_{n+1}\right), \bar{x}-x_{n+1}\right\rangle,
$$

which ensures with (65) that

$$
\phi_{n+1}-\phi_{n} \leq \lambda_{n}(2 \rho-\sigma) W\left(x_{n+1}, \bar{x}\right) .
$$

Using the assumption $\sigma \geq 2 \rho$ yields

$$
\phi_{n+1} \leq \phi_{n} .
$$

Following the same reasoning in the proof of Theorem 14 and the ball compactness of the image of $C$, we get a subsequence $\left\{x_{n_{k}}\right\}$ which converges to some limit $\widetilde{x}$ satisfying $\tilde{x} \in C(\tilde{x})$ by closedness of the graph of $C$. By Algorithm 15 this subsequence satisfies

$$
\begin{aligned}
\left\langle x_{n_{k}+1}^{*}, x-x_{n_{k}+1}\right\rangle \leq & F\left(x_{n_{k}+1}, x\right) \\
& +\rho V\left(J\left(x_{n_{k}+1}\right), x\right), \\
& \forall k, \forall x \in \operatorname{Im} C .
\end{aligned}
$$

Thus, by letting $k \rightarrow \infty$ in the inequality (69) and by taking into account the upper semicontinuity of $F$ and the continuity of $V$ and $J$, we obtain

$$
0 \leq F(\widetilde{x}, x)+\rho V(J(\widetilde{x}), x), \quad \forall x \in C(\widetilde{x}) .
$$

This means that $\tilde{x}$ is a solution of $(\operatorname{NQEP}[C, F])$ which ensures by Proposition 12 that under the assumptions of our theorem the solution $\tilde{x}$ is also a solution of $(\operatorname{NQVP}[C, F])$. Thus completing the proof.

4.3. Case 3: $F$ Has the Form: $F(x, y)=\langle T(x), y-x\rangle$. In this subsection we restrict our attention to the following form of the bifunction $F$ :

$$
F(x, y)=\langle T(x), y-x\rangle,
$$

where $T: X \rightarrow X^{*}$ is a nonlinear operator. In this case $\partial^{\pi} F(\bar{x}, \cdot)(\bar{x})=\{T(\bar{x})\}$ and so (NQVP $\left.[C, F]\right)$ becomes:

Find $\bar{x} \in C(\bar{x})$,

such that $T(\bar{x}) \in-N^{\pi}(C(\bar{x}), \bar{x})$.

$(\mathrm{NQVP}[C, T])$

We suggest the following algorithm to solve (NQVP $[C, T])$ under some natural and appropriate assumptions on $C$ and $T$.
Algorithm 17. Let $\delta_{n} \downarrow 0$ with $\delta_{0}$ be too small.

(i) Select $x_{0} \in C\left(x_{0}\right), y_{0}^{*}=T\left(x_{0}\right)$ and $\rho>0$;

(ii) For $n \geq 0$,

(a) Compute $z_{n+1}:=J^{*}\left(J\left(x_{n}\right)-\rho y_{n}^{*}\right)$;

(b) Compute $x_{n+1}:=\pi_{C\left(x_{n}\right)}\left(J\left(z_{n+1}\right)\right)$ and $y_{n+1}^{*}:=$ $T\left(x_{n+1}\right)$,

where $\pi_{S}$ is the generalised projection defined in terms of the functional $V$ instead of the norm square (introduced and studied in the convex case in [16] and for the nonconvex case we refer to the recent paper [11]). A point $\bar{x} \in S$ is called the generalised projection of a given $x^{*} \in X^{*}$ provided that

$$
V\left(x^{*}, \bar{x}\right)=\inf _{s \in S} V\left(x^{*}, s\right) .
$$

The following characterisation of the $V$-proximal normal cone in terms of the generalised projection is proved in [1].

Proposition 18. For any closed nonempty set $S$ in a reflexive Banach space $X$ and for any point $\bar{x} \in S$ we have

$$
\begin{aligned}
& N^{\pi}(S ; \bar{x})=\left\{x^{*} \in X^{*}: \exists \lambda>0 \text { such that } \bar{x}\right. \\
& \left.\quad \in \pi_{S}\left(J(\bar{x})+\lambda x^{*}\right)\right\} .
\end{aligned}
$$

We need the following lemma:

Lemma 19. Let $S$ be a closed set in $X, \bar{x} \in S, y^{*} \in X^{*}$, and $r>0$. If $\bar{x} \in \pi_{S}\left(J(\bar{x})-r y^{*}\right)$; then $\bar{x} \in \pi_{S}\left(J(\bar{x})-\rho y^{*}\right)$, for any $\rho \in[0, r]$.

Proof. Let $r>0, y^{*} \in X^{*}$, and let $\bar{x}$ be a point satisfying $\bar{x} \epsilon$ $\pi_{S}\left(J(\bar{x})-r y^{*}\right)$. Assume that $\rho \in[0, r]$. Let $\lambda:=\rho / r \in[0,1]$. We claim that

$$
V\left(J(\bar{x})-\rho y^{*}, \bar{x}\right)=\inf _{s \in S} V\left(J(\bar{x})-\rho y^{*}, s\right) .
$$

First, observe that for any $s \in S$ we have

$$
\begin{aligned}
2\left\langle J(\bar{x})-\rho y^{*}-J \bar{x} ; s-\bar{x}\right\rangle \\
\quad=2\left\langle\lambda\left(J(\bar{x})-r y^{*}\right)+(1-\lambda) J(\bar{x})-J \bar{x} ; s-\bar{x}\right\rangle \\
\quad=2 \lambda\left\langle\left(J(\bar{x})-r y^{*}\right)-J(\bar{x}) ; s-\bar{x}\right\rangle .
\end{aligned}
$$

If $\left\langle\left(J(\bar{x})-r y^{*}\right)-J(\bar{x}) ; s-\bar{x}\right\rangle<0$, then obviously we have

$$
2\left\langle J(\bar{x})-\rho y^{*}-J \bar{x} ; s-\bar{x}\right\rangle<0 \leq V(J(\bar{x}), s) .
$$

Otherwise, we have $\left\langle\left(J(\bar{x})-r y^{*}\right)-J(\bar{x}) ; s-\bar{x}\right\rangle \geq 0$. Then since $0 \leq \lambda \leq 1$ we have

$$
\begin{aligned}
& 2 \lambda\left\langle\left(J(\bar{x})-r y^{*}\right)-J(\bar{x}) ; s-\bar{x}\right\rangle \\
& \quad \leq 2\left\langle\left(J(\bar{x})-r y^{*}\right)-J(\bar{x}) ; s-\bar{x}\right\rangle
\end{aligned}
$$


and so we obtain

$$
\begin{aligned}
& 2\left\langle J(\bar{x})-\rho y^{*}-J \bar{x} ; s-\bar{x}\right\rangle \\
& \leq 2\left\langle\left(J(\bar{x})-r y^{*}\right)-J(\bar{x}) ; s-\bar{x}\right\rangle \\
& \leq\left\|J(\bar{x})-r y^{*}\right\|^{2}-2\left\langle\left(J(\bar{x})-r y^{*}\right) ; \bar{x}\right\rangle+\|\bar{x}\|^{2} \\
&+2\left\langle\left(J(\bar{x})-r y^{*}\right) ; s\right\rangle-\left\|J(\bar{x})-r y^{*}\right\|^{2}-\|s\|^{2} \\
&+\|s\|^{2}-2\langle J(\bar{x}) ; s-\bar{x}\rangle-\|\bar{x}\|^{2} \\
& \leq V\left(J(\bar{x})-r y^{*}, \bar{x}\right)-V\left(J(\bar{x})-r y^{*}, s\right) \\
&+V(J(\bar{x}), s) \\
& \leq \inf _{z \in S} V\left(J(\bar{x})-r y^{*}, z\right)-V\left(J(\bar{x})-r y^{*}, s\right) \\
&+V(J(\bar{x}), s) \leq V(J(\bar{x}), s) ;
\end{aligned}
$$

that is,

$$
2\left\langle J(\bar{x})-\rho y^{*}-J \bar{x} ; s-\bar{x}\right\rangle \leq V(J(\bar{x}), s) .
$$

Therefore, from (76) and (79) we have in both cases

$$
2\left\langle J(\bar{x})-\rho y^{*}-J \bar{x} ; s-\bar{x}\right\rangle \leq V(J(\bar{x}), s), \quad \forall s \in S .
$$

Hence

$$
2\left\langle J(\bar{x})-\rho y^{*}-J \bar{x} ; s-\bar{x}\right\rangle-V(J(\bar{x}), s) \leq 0,
$$

$$
\forall s \in S \text {. }
$$

On the other hand we have the decomposition

$$
\begin{aligned}
& 2\left\langle J(\bar{x})-\rho y^{*}-J \bar{x} ; s-\bar{x}\right\rangle-V(J(\bar{x}), s) \\
&=2\left\langle J(\bar{x})-\rho y^{*} ; s\right\rangle-2\left\langle J(\bar{x})-\rho y^{*} ; \bar{x}\right\rangle+2\|\bar{x}\|^{2} \\
&-2\langle J \bar{x} ; s\rangle-\left[\|\bar{x}\|^{2}-2\langle J \bar{x} ; s\rangle+\|s\|^{2}\right] \\
&= {\left[\left\|J(\bar{x})-\rho y^{*}\right\|^{2}-2\left\langle J(\bar{x})-\rho y^{*} ; \bar{x}\right\rangle+\|\bar{x}\|^{2}\right] } \\
&-\left[\left\|J(\bar{x})-\rho y^{*}\right\|^{2}-2\left\langle J(\bar{x})-\rho y^{*} ; s\right\rangle+\|s\|^{2}\right] \\
&= V\left(J(\bar{x})-\rho y^{*}, \bar{x}\right)-V\left(J(\bar{x})-\rho y^{*}, s\right) .
\end{aligned}
$$

Consequently, we have

$$
\begin{array}{r}
V\left(J(\bar{x})-\rho y^{*}, \bar{x}\right)-V\left(J(\bar{x})-\rho y^{*}, s\right) \leq 0, \\
\text { for any } s \in S,
\end{array}
$$

that is,

$$
V\left(J(\bar{x})-\rho y^{*}, \bar{x}\right)=\inf _{s \in S} V\left(J(\bar{x})-\rho y^{*}, s\right) ;
$$

which means that $\bar{x} \in \pi_{S}\left(J(\bar{x})-\rho y^{*}\right)$ and hence the proof is complete.

Now, we state and prove our main theorem for $(\mathrm{NQVP}[C, T])$.
Theorem 20. Let $X$ be a 2-uniformly smooth Banach space. Let $C: X \rightrightarrows X$ be a set-valued mapping with closed nonempty values and $T: X \rightarrow X^{*}$. Let $\left\{x_{n}\right\}_{n}$ be a sequence generated by Algorithm 17. Assume that

(1) The solution set of (NQVP[C,T]) is nonempty;

(2) $T$ is bounded by some constant $L>0$;

(3) $T$ is J-Lipschitz, with constant $\beta>0$; that is,

$\left\|T\left(x_{1}\right)-T\left(x_{2}\right)\right\| \leq \beta\left\|J\left(x_{1}\right)-J\left(x_{2}\right)\right\|$,

$$
\forall x_{i} \in X, i=1,2 \text {; }
$$

(4) $T$ is J-strongly monotone with constant $\alpha>0$; that is,

$$
\begin{aligned}
& \left\langle J^{*}\left(T\left(x_{1}\right)-T\left(x_{2}\right)\right) ; J\left(x_{1}\right)-J\left(x_{2}\right)\right\rangle \\
& \quad \geq \alpha\left\|J\left(x_{1}\right)-J\left(x_{2}\right)\right\|^{2}, \quad \forall x_{1}, x_{2} \in X ;
\end{aligned}
$$

(5) The values of $C$ satisfy for some $r \in(0, \infty]$ :

$$
\bar{u} \in \pi_{C(\bar{u})}\left(J(\bar{u})+r u^{*}\right), \quad \forall u^{*} \in X^{*}
$$

for any unit vector $u^{*}$ in $X^{*}$ and any $\bar{u}$ solution of (NQVP $[C, T])$;

(6) There exists some constant $k \in(0,1)$ and $\xi>0$ such that

$$
\begin{aligned}
& \left\|J\left(\pi_{C\left(x_{1}\right)}\left(x_{1}^{*}\right)\right)-J\left(\pi_{C\left(x_{2}\right)}\left(x_{2}^{*}\right)\right)\right\| \\
& \quad \leq \xi\left\|x_{1}^{*}-x_{2}^{*}\right\|+k\left\|J\left(x_{1}\right)-J\left(x_{2}\right)\right\|,
\end{aligned}
$$

for all $x_{i} \in X, x_{i}^{*} \in X^{*}, i=1,2$;

(7) The positive constants $\alpha$ and $\beta$ satisfy the inequality $\alpha>$ $\beta \sqrt{1-(1-k)^{2} / c \xi^{2}}$

(8) The parameter $\rho$ in Algorithm 17 satisfies

$$
\begin{aligned}
\frac{\alpha}{\beta^{2}}-\bar{\epsilon} & <\rho<\min \left\{\frac{\mu-\delta_{0}}{L}, \frac{\alpha}{\beta^{2}}+\bar{\epsilon}\right\}, \\
\bar{\epsilon} & :=\frac{\sqrt{\alpha^{2}-\beta^{2}\left(1-(1-k)^{2} / c \xi^{2}\right)}}{\beta^{2}} .
\end{aligned}
$$

Then, the sequence $\left\{x_{n}\right\}_{n}$ generated by Algorithm 17 converges to a solution of (NQVP[C,T]).

Proof. Let $\bar{x} \in C(\bar{x})$ be a solution of (NQVP $[C, T])$, that is, $-T(\bar{x}) \in N^{\pi}(C(\bar{x}) ; \bar{x})$. Then by the characterisation of the $V-$ proximal normal cone in Proposition 18, there exists $\lambda>0$ such that $\bar{x} \in \pi_{C(\bar{x})}(J(\bar{x})-\lambda T(\bar{x}))$. Using Lemma 19 we obtain $\bar{x} \in \pi_{C(\bar{x})}(J(\bar{x})-\tau T(\bar{x}))$, for any $\tau \in[0, \lambda]$. By assumption (5) we may assume that $\lambda \leq r / L$ and so we get $\rho \leq r / L$. Hence $\bar{x} \epsilon$ $\pi_{C(\bar{x})}(J(\bar{z}))$ for $\bar{z}:=J^{*}(J(\bar{x})-\rho T(\bar{x}))$. Since $X$ is 2-uniformly smooth we have $X^{*}$ is 2-uniformly convex; that is,

$$
\delta_{X^{*}}(\epsilon) \geq 2 c^{-1} \epsilon^{2},
$$


for some constant $c>0$ (depending only on the space $X^{*}$ ) and so by Lemma 7 we get

$$
\begin{aligned}
V_{*}\left(J^{*} x^{*}, y^{*}\right) & \geq 8 C^{2} \delta_{X^{*}}\left(\frac{\left\|x^{*}-y^{*}\right\|}{4 C}\right) \\
& \geq c^{-1}\left\|x^{*}-y^{*}\right\|^{2}, \quad \forall x^{*}, y^{*} \in X^{*}
\end{aligned}
$$

Thus we can write

$$
\begin{aligned}
\| \rho & {\left[T\left(x_{n}\right)-T(\bar{x})\right]-\left(J\left(x_{n}\right)-J(\bar{x})\right) \|^{2} } \\
& \leq c\left[V_{*}\left(\rho J^{*}\left(T\left(x_{n}\right)-T(\bar{x})\right) ; J\left(x_{n}\right)-J(\bar{x})\right)\right] .
\end{aligned}
$$

Therefore,

$$
\begin{aligned}
& \left\|J\left(z_{n+1}\right)-J(\bar{z})\right\|^{2} \\
& =\left\|J\left(x_{n}\right)-\rho T\left(x_{n}\right)-J(\bar{x})+\rho T(\bar{x})\right\|^{2} \\
& \leq c\left[V_{*}\left(\rho J^{*}\left(T\left(x_{n}\right)-T(\bar{x})\right) ; J\left(x_{n}\right)-J(\bar{x})\right)\right] \\
& \leq c\left[\rho^{2}\left\|T\left(x_{n}\right)-T(\bar{x})\right\|^{2}+\left\|J\left(x_{n}\right)-J(\bar{x})\right\|^{2}\right] \\
& \quad-2 c \rho\left\langle J^{*}\left(T\left(x_{n}\right)-T(\bar{x})\right) ; J\left(x_{n}\right)-J(\bar{x})\right\rangle .
\end{aligned}
$$

Using the $J$-Lipschitz continuity of $T$ with ratio $\beta$ we have

$$
\left\|T\left(x_{n}\right)-T(\bar{x})\right\|^{2} \leq \beta^{2}\left\|J\left(x_{n}\right)-J(\bar{x})\right\|^{2}
$$

and by the $J$-strong monotonicity of $T$ with ratio $\alpha$ we have

$$
\begin{aligned}
& \left\langle J^{*}\left(T\left(x_{n}\right)-T(\bar{x})\right) ; J\left(x_{n}\right)-J(\bar{x})\right\rangle \\
& \quad \geq \alpha\left\|J\left(x_{n}\right)-J(\bar{x})\right\|^{2} .
\end{aligned}
$$

Thus, we get

$$
\begin{aligned}
& \left\|J\left(z_{n+1}\right)-J(\bar{z})\right\|^{2} \\
& \leq c\left[\rho^{2} \beta^{2}\left\|J\left(x_{n}\right)-J(\bar{x})\right\|^{2}+\left\|J\left(x_{n}\right)-J(\bar{x})\right\|^{2}\right] \\
& \quad-2 c \rho \alpha\left\|J\left(x_{n}\right)-J(\bar{x})\right\|^{2} \\
& \leq c\left(1+\rho^{2} \beta^{2}-2 \rho \alpha\right)\left\|J\left(x_{n}\right)-J(\bar{x})\right\|^{2}
\end{aligned}
$$

and so

$$
\begin{aligned}
& \left\|J\left(z_{n+1}\right)-J(\bar{z})\right\| \\
& \quad \leq \sqrt{c\left(1+\rho^{2} \beta^{2}-2 \rho \alpha\right)}\left\|J\left(x_{n}\right)-J(\bar{x})\right\| .
\end{aligned}
$$

On the other hand we have by the 6th assumption

$$
\begin{aligned}
\| J & \left(x_{n+1}\right)-J(\bar{x}) \| \\
& =\left\|J\left(\pi_{C\left(x_{n}\right)}\left(J\left(z_{n+1}\right)\right)\right)-J\left(\pi_{C(\bar{x})}(J(\bar{z}))\right)\right\| \\
& \leq \xi\left\|J\left(z_{n+1}\right)-J(\bar{z})\right\|+k\left\|J\left(x_{n}\right)-J(\bar{x})\right\| .
\end{aligned}
$$

Thus

$$
\begin{aligned}
& \left\|J\left(x_{n+1}\right)-J(\bar{x})\right\| \\
& \quad \leq\left(k+\xi \sqrt{c\left(1+\rho^{2} \beta^{2}-2 \rho \alpha\right)}\right)\left\|J\left(x_{n}\right)-J(\bar{x})\right\| .
\end{aligned}
$$

Our assumptions and the choice of $\rho$ ensure that $(k+$ $\left.\xi \sqrt{c\left(1+\rho^{2} \beta^{2}-2 \rho \alpha\right)}\right)<1$ and hence $\left\|J\left(x_{n}\right)-J(\bar{x})\right\| \rightarrow 0$ which means that $x_{n} \rightarrow \bar{x}$ by the uniform continuity of $J^{*}$ and thus completing the proof.

Remark 21. A simple inspection of the proof of the previous theorem shows that the result is valid in the case when $T$ is taken a general set-valued mapping instead of a single-valued operator defined from $X$ to $X^{*}$ and of course the assumptions on $T$ should be adapted naturally for the set-valued case.

\section{Competing Interests}

The authors declare that there are no competing interests regarding the publication of this paper.

\section{Acknowledgments}

This research was supported for the first author by the NSTIP Strategic Technologies Program in the Kingdom of Saudi Arabia, Award no. (11-MAT1916-02).

\section{References}

[1] M. Bounkhel and R. Al-Yusof, "Proximal analysis in reflexive smooth Banach spaces," Nonlinear Analysis: Theory, Methods of Applications, vol. 73, no. 7, pp. 1921-1939, 2010.

[2] M. Bounkhel, L. Tadj, and A. Hamdi, "Iterative schemes to solve nonconvex variational problems," Journal of Inequalities in Pure and Applied Mathematics, vol. 4, no. 1, article no. 14, p. 14, 2003.

[3] M. Bounkhel and J. Bounkhel, "Nonconvex variational inequalities," ESAIM: Control, Optimisation and Calculus of Variations, vol. 11, no. 4, pp. 574-594, 2005.

[4] M. A. Noor, "Iterative schemes for nonconvex variational inequalities," Journal of Optimization Theory and Applications, vol. 121, no. 2, pp. 385-395, 2004.

[5] M. A. Noor and K. I. Noor, "On equilibrium problems," Applied Mathematics E-Notes, vol. 4, pp. 125-132, 2004.

[6] M. A. Noor, K. I. Noor, and S. Zainab, "Some iterative methods for solving nonconvex bifunction equilibrium variational inequalities," Journal of Applied Mathematics, vol. 2012, Article ID 280451, 10 pages, 2012.

[7] A. Moudafi; "Second-order differential proximal methods for Equilbrium problems," Journal of Inequalities in Pure and Applied Mathematics, vol. 4, no. 1, article 15, 2003.

[8] M. Bounkhel and B. R. Al-Sinan, "An iterative method for nonconvex equilibrium problems," Journal of Inequalities in Pure and Applied Mathematics, vol. 7, no. 2, article 75, 8 pages, 2006.

[9] G. Stampacchia, "Formes Bilinéaires coercives sur les ensembles convexes," Compte Rendus de l'Acadmie des Sciences, Paris, vol. 258, pp. 4413-4416, 1964. 
[10] R. Deville, G. Godefroy, and V. Zizler, Smoothness and Renormings in Banach spaces, Pitman Monographs and Surveys in Pure and Applied Mathematics, Longman Scientific \& Technical, Harlow, UK, 1993.

[11] M. Bounkhel, "Calculus rules for $V$-proximal subdifferentials in smooth Banach spaces," Journal of Function Spaces, vol. 2016, Article ID 1917387, 12 pages, 2016.

[12] R. Deville, R. Gonzalo, and J. A. Jaramilo, "Renormings of Lp(Lq)," Mathematical Proceedings of the Cambridge Philosophical Society, vol. 126, no. 1, pp. 155-169, 1999.

[13] M. Bounkhel, "Iterative Methods for nonconvex equilibrium problems in Banach spaces," Journal of Function Spaces, vol. 2015, Article ID 346830, 10 pages, 2015.

[14] F. H. Clarke, R. J. Stern, and P. R. Wolenski, "Proximal smoothness and the lower C2 property," Journal of Convex Analysis, vol. 2, no. 1-2, pp. 117-144, 1995.

[15] R. A. Poliquin, R. T. Rockafellar, and L. Thibault, "Local differentiability of distance functions," Transactions of the American Mathematical Society, vol. 352, no. 11, pp. 5231-5249, 2000.

[16] Y. I. Alber, "Generalized projection operators in Banach spaces: properties and applications," Functional Differential Equations, vol. 1, no. 1, pp. 1-21, 1994.

[17] M. Bounkhel, Regularity Concepts in Nonsmooth Analysis, Theory and Applications, vol. 59 of Springer Optimization and Its Applications, Springer, New York, NY, USA, 2012. 


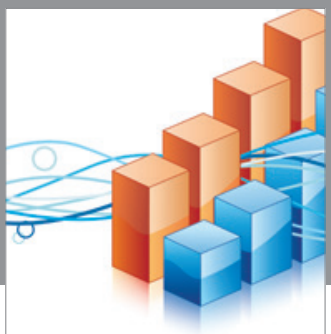

Advances in

Operations Research

vatem alat4

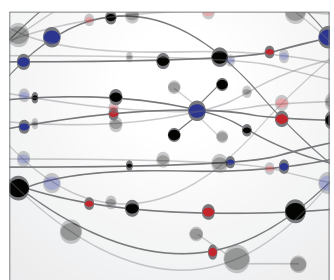

\section{The Scientific} World Journal
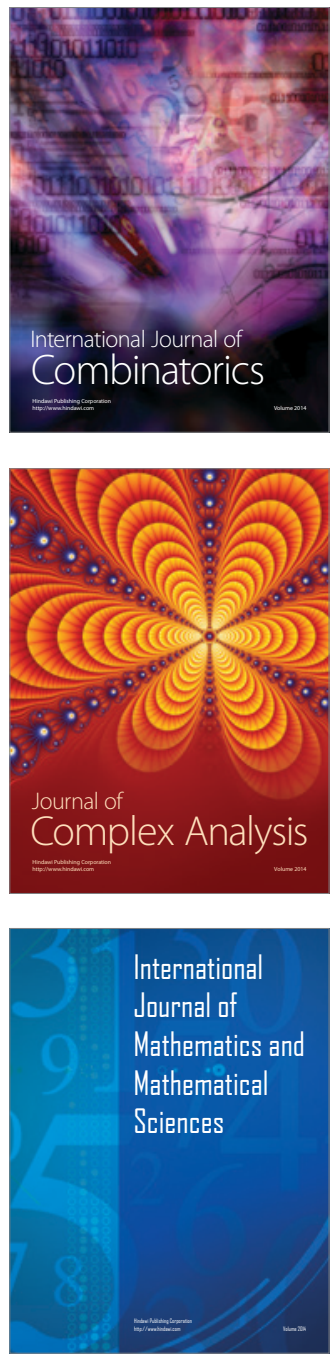
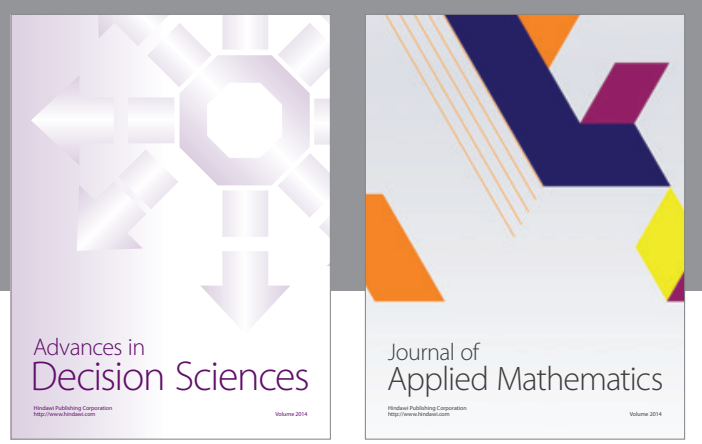

Algebra

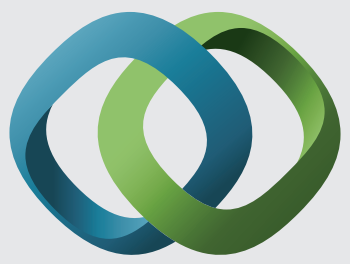

\section{Hindawi}

Submit your manuscripts at

https://www.hindawi.com
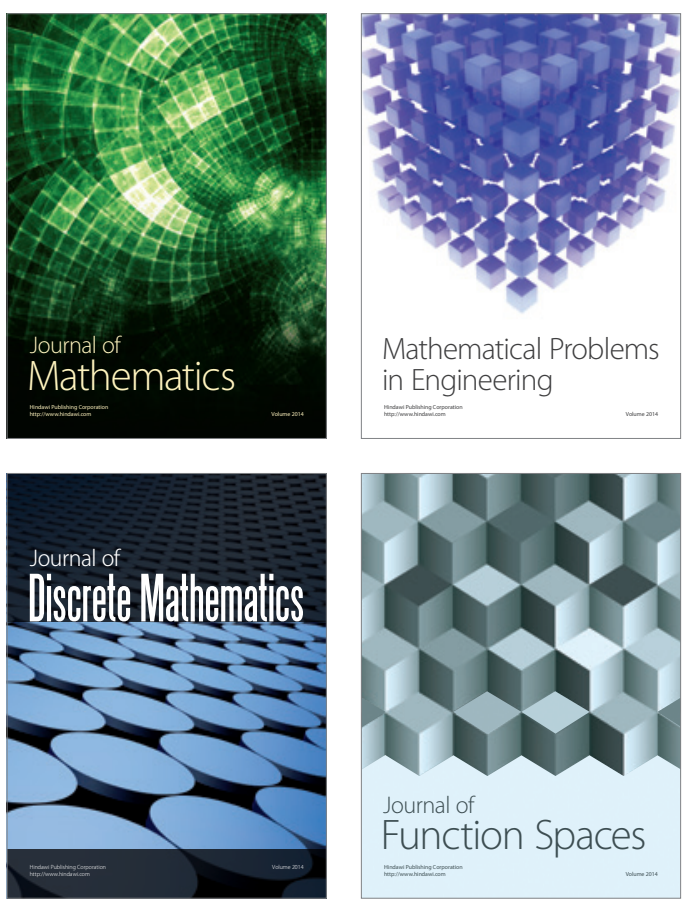

Mathematical Problems in Engineering
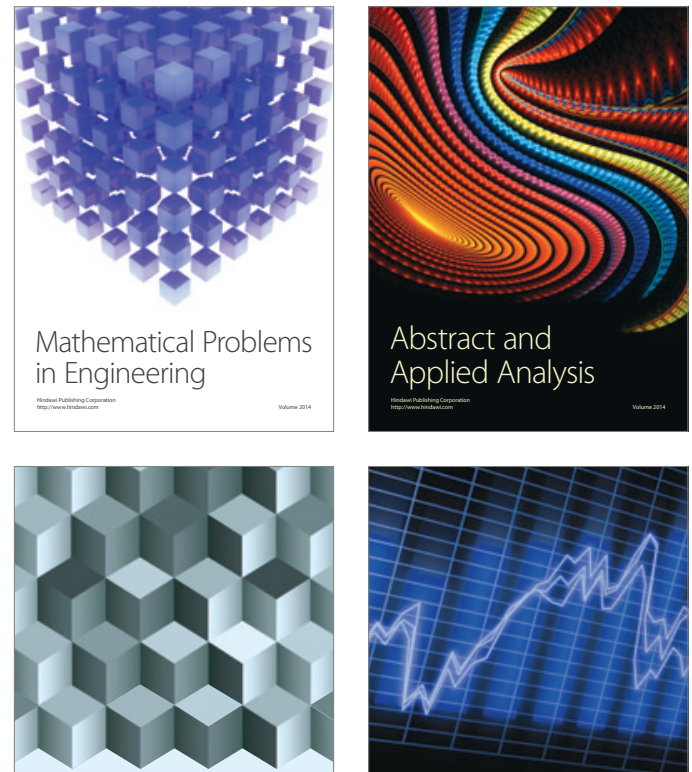

Journal of

Function Spaces

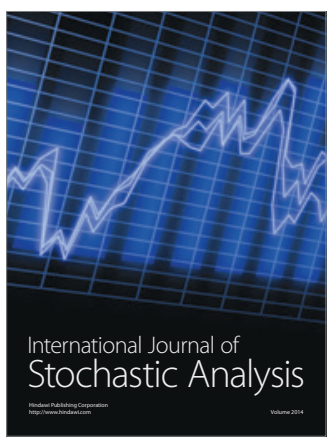

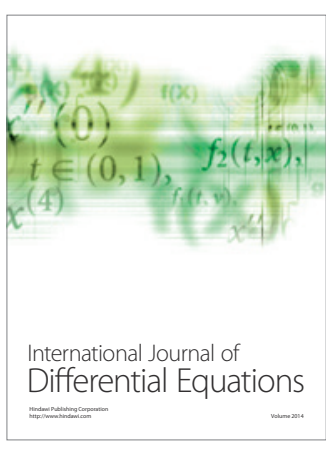
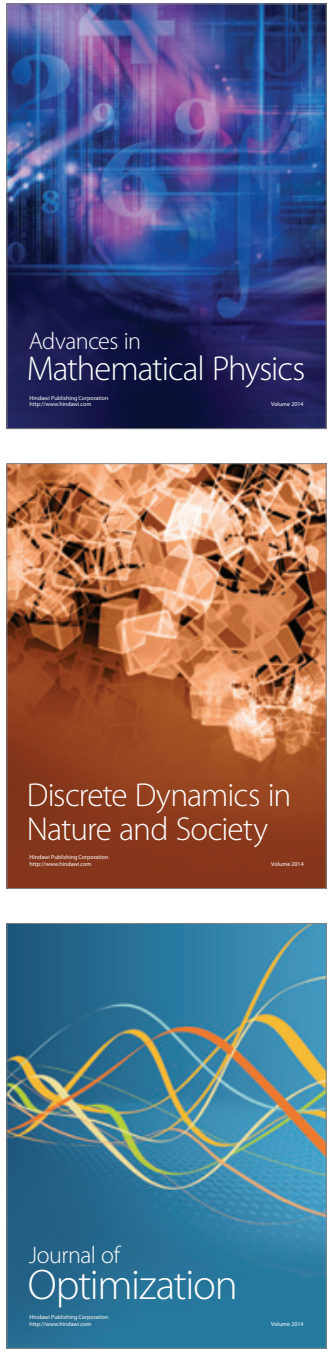